\title{
Effect of bright light treatment on agitated behavior in institutionalized elderly subjects
}

\author{
Barbara B. Lovell ${ }^{\mathrm{a}}$, Sonia Ancoli-Israel* ${ }^{\mathrm{b}}$, Richard Gevirtz ${ }^{\mathrm{a}}$ \\ ${ }^{a}$ California School of Professional Psychology, San Diego, CA 92121, USA \\ ${ }^{b}$ Department of Psychiatry (II6A), University of California San Diego, Veterans Affairs Medical Center, 3350 La Jolla Village \\ Drive, San Diego, CA 92161, USA
}

Received 28 September 1993; revision received 15 March 1994; accepted 3 May 1994

\begin{abstract}
This study examined whether exposure to bright light treatment would reduce agitated behavior in institutionalized elderly subjects. Six demented elderly subjects (mean age $=\mathbf{8 9 . 2}$ years) living in a skilled nursing facility were studied. Light (2500 lx) was administered for 2 hours in the morning for two 10-day periods. The Bliwise Agitation Behavior Rating Scale was used to rate agitated behavior once every $15 \mathrm{~min}$ between 16:00 $\mathrm{h}$ and 20:00 $\mathrm{h}$ during 3 days of baseline, the light treatment periods, and 5 days of posttreatment follow-up evaluation. The entire protocol was then repeated in an ABABA design. A planned comparison revealed a significant difference between light treatment days and nontreatment days, with less agitation being observed on treatment days. The study suggests the efficacy of the clinical use of bright light treatment to reduce agitation.
\end{abstract}

Keywords: Dementia; Sundowning; Geriatric psychiatry; Nursing home residents

\section{Introduction}

Agitated behavior is very common in dementia and is one of the hallmarks of the demented patient (Jackson et al., 1987; Wild et al., 1987; Cohen-Mansfield et al., 1990; Jeste and Krull, 1991; Pollack and Perlick, 1991; Aronson et al., 1992). Until recently, studies on agitation in institutionalized elderly subjects were sparse and primarily anecdotal, but data suggest a connection

* Corresponding author, Tel: +1 619 552-8585, ext. 3828; Fax: +1619 552-7536. between dementia and/or brain damage and agitation (Chesrow et al., 1965; Granacher, 1982; Fisher et al., 1983).

Agitation is not a diagnostic term; rather it is used to describe a group of symptoms. CohenMansfield and colleagues defined symptoms of agitation as aggressive, nonaggressive, or verbal behaviors that are manifested as increased confusion, wandering, attempts to escape restraints, calling out, or screaming (Cohen-Mansfield, 1986; Cohen-Mansfield and Billig, 1986; CohenMansfield et al., 1989a, 1989b, 1990). Agitation is extremely common in nursing home patients and 
poses a challenge for nursing staff who must care for the agitated patient while simultaneously attempting to calm other patients disturbed by the noise or activity. Currently, problems of agitation are managed by using medications, restraints, or environmental design changes or by increasing the staff-to-resident ratio (Cohen-Mansfield et al., 1989a).

Agitated behavior in some elderly nursing home residents increases as the sun sets. This phenomenon is referred to as the sundown syndrome. Evans (1987) reported that $12.3 \%$ of institutionalized elderly were more inappropriately restless and verbal in the late afternoon. Cameron (1941) reported that senile nocturnal delirium could be precipitated by placing an elderly demented patient in a darkened room during the daytime. CohenMansfield and colleagues (Cohen-Mansfield, 1986; Cohen-Mansfield et al., 1989a) observed the frequency and time of patients' agitated behavior over a 24-h day and detected temporal patterns of disturbance, with some residents being more agitated in the mornings and some in the evenings.

Clinicians do not yet know what causes, prevents, or alleviates agitation or sundowning behavior. Bliwise et al. (1989a, 1993) found seasonal variation in agitated behavior, with increased agitation occurring in the darker winter months. If darkness precipitates agitation, then light treatment may be a promising alternative to current practices. Studies in Japan found that both morning and evening bright light treatment was effective in decreasing agitated behavior (Okawa et al., 1989; Hozumi et al., 1990). Satlin et al. (1992) studied the effects of bright light treatment on 10 patients with Alzheimer's disease who were characterized by agitated behavior and sleep disturbances. Sundowning scores were significantly reduced after 1 week of evening bright light treatment. Those patients with the most severe sundowning behaviors at baseline improved the most during the treatment and posttreatment week. The amplitude of the circadian rest-activity cycle increased with light treatment, also suggesting that sundowning may be associated with disturbed circadian rhythms. The results of this study were limited by the lack of a control group and the fact that light treatment was not reintroduced following the posttreatment week.
In summary, agitation is a prevalent and important problem. To date, no treatments have been totally satisfactory in effectively managing the disturbances. Medications are limited by side effects, restraints do not adequately solve the problem, psychotherapy is only partially effective and often unavailable, and environmental changes frequently cannot be made. Controlled studies looking at the relationship between dementia, agitation, and bright light in the skilled nursing home population are still needed. Thus, the objective of the present study was to examine whether agitated behavior in the institutionalized elderly could be effectively treated with bright light treatment.

\section{Methods}

\subsection{Subjects}

Five women and one man, residents of the Hebrew Home for the Aged in San Diego, California, participated in the study. All were Caucasians, who ranged in age from 85 to 95 years (mean age $=89.2$ years). Subjects had lived on the skilled care wing in the nursing home for at least 3 months, had some degree of agitation, and were judged by nursing staff to be adjusted to institutional living. All were moderately to severely demented and mildly depressed. None of the residents were legally blind, and all were receiving medications (see Table 1).

\subsection{Apparatus}

A 2500 lux light box ( 2 feet $\times 1$ foot), containing cool-white fluorescent bulbs, was used ("Brite Lite" Apollo Light Systems, Orem, Utah). The box was shielded to limit ultraviolet and radiofrequency radiations, and contained a built-in ballast that augmented the light's brightness.

Agitated behaviors were assessed with the Agitation Behavior Rating Scale (ABRS), a timebased, behavior-specific measure that was used repeatedly throughout the day (Bliwise et al., 1989a, 1989b). The scale rates five behaviors: (a) manual manipulation, (b) escape restraints, (c) searching and wandering, (d) tapping and banging, and (e) vocalization. A 4-point scale of intensity of agitation was used $(0=$ none, $1=$ mild, $2=$ moderate, and $3=$ severe). The ABRS has been shown to be reliable in a nursing home for the 
Table 1

Medical history and demographics

\begin{tabular}{|c|c|c|c|c|c|c|}
\hline Subject No. & Age & Gender & MMSE & GDS & Diagnoses & Medications \\
\hline 1 & 87 & Male & 7 & 15 & $\begin{array}{l}\text { Cardiovascular, renal, } \\
\text { endocrine, dementia }\end{array}$ & Insulin, aspirin \\
\hline 2 & 95 & Female & 0 & 12 & $\begin{array}{l}\text { Cardiovascular, connective } \\
\text { tissue, dementia }\end{array}$ & Digoxin, furosemide \\
\hline 3 & 90 & Female & 14 & 12 & Cardiovascular, endocrine & $\begin{array}{l}\text { Metoclopramide, } \\
\text { haloperidol, } \\
\text { nitroglyccrin, ferrous } \\
\text { sulfate }\end{array}$ \\
\hline 4 & 90 & Female & 14 & 3 & $\begin{array}{l}\text { Cental nervous system, } \\
\text { endocrine }\end{array}$ & $\begin{array}{l}\text { Antacids, anti- } \\
\text { Parkinson }\end{array}$ \\
\hline 5 & 88 & Female & 9 & 9 & $\begin{array}{l}\text { Malignancies, gastro- } \\
\text { intestinal, endocrine }\end{array}$ & $\begin{array}{l}\text { Metoclopramide, } \\
\text { osmolite }\end{array}$ \\
\hline 6 & 85 & Female & 8 & 10 & $\begin{array}{l}\text { Gastrointestinal, endocrine, } \\
\text { dementia }\end{array}$ & $\begin{array}{l}\text { Antidepressants, } \\
\text { haloperidol, thyroid } \\
\text { hormone, ferrous } \\
\text { gluconate }\end{array}$ \\
\hline
\end{tabular}

Note. MMSE, Mini-Mental State Examination. GDS, Geriatric Depression Scale. GDS scores: 0-9, normal; 10-15, mild depression; and $15-20$, severe depression.

assessment of agitated behavior, with percentage of agreement between observers ranging from 80.16 to $96.83 \%$ (Bliwise et al., 1989a).

\subsection{Procedure}

The subjects were all participants in a larger study on sleep. Those who were agitated and were being exposed to morning light, as part of the sleep study, were also enrolled in the present study. Written informed consent from the subject or the subject's guardian and verbal consent from the subject's physician were obtained. A copy of the consent form, which explained the entire study, and a copy of the experimental subject's bill of rights was given to each subject and/or guardian.

Four subjects participated in a 36-day protocol: (a) 3 days of baseline 1, (b) 10 days of morning bright light treatment 1 , (c) 5 days of posttreatment follow-up 1, (d) 3 days of baseline 2, (e) 10 days of morning bright light treatment 2 , and (f) 5 days of posttreatment follow-up 2 . The remaining two subjects lacked 3 days of baseline 2 and had only 5 days of light treatment 2 . Competing needs of equipment for the main sleep study created this discrepancy.
Light ( 2500 lux) was administered between 09:30 and 11:30 h on each treatment day. Treatment took place wherever the subject was most comfortable, usually in a chair by the side of the bed. The light box was placed approximately $1 \mathrm{~m}$ away from the patient at a height within the patient's visual field. Light was checked by photometer, which was held at the level of the subject's eyes, at the beginning, middle, and end of treatment. Subjects were encouraged to watch television or enjoy any normal activity during the treatment period.

Behaviors were observed $4 \mathrm{~h}$ a day at 15 -min intervals from 16:00 to $20: 00 \mathrm{~h}$. The five behaviors of the ABRS were scored during each of the observations. At the end of the 4-h observation period, all scores were totaled, and the sum of the scores became the agitated behavior score for the day. Observations were made by one of five observers with an interrater percentage of agreement that ranged from 96 to $99 \%$. All raters observed the subjects for signs of agitation both in this protocol and in the sleep protocol (which involved treatments other than morning bright light) and were unaware of this study's hypothesis about morning bright light therapy. 
Table 2

Individual agitation scores

\begin{tabular}{|c|c|c|c|c|c|c|}
\hline Subject No. & $A^{a}$ & B & $\mathrm{C}$ & $\mathrm{D}$ & $\mathrm{E}$ & $\mathbf{F}$ \\
\hline 1 & 34.6 & 8.3 & 20.8 & $20.8^{b}$ & 8.6 & 28.5 \\
\hline 2 & 39.5 & 14.0 & 26.3 & $26.3^{b}$ & 20.0 & 46.3 \\
\hline 3 & 6.3 & 11.8 & 9.8 & 14.8 & 7.3 & 6.6 \\
\hline 4 & 7.8 & 5.3 & 5.0 & 25.7 & 3.8 & 10.8 \\
\hline 5 & 17.7 & 4.0 & 13.8 & 10.7 & 3.5 & 4.4 \\
\hline 6 & 14.3 & 19.8 & 26.2 & 20.7 & 10.2 & 31.8 \\
\hline Mean $^{c}$ & 20.03 & 10.53 & 16.98 & 19.83 & 8.90 & 21.40 \\
\hline SD & 13.90 & 5.91 & 8.85 & 6.11 & 6.05 & 16.73 \\
\hline
\end{tabular}

${ }^{a} A$, baseline 1; B, treatment 1 ; C, posttreatment 1 ; D, baseline 2; E, treatment 2; F, posttreatment 2.

${ }^{b}$ Baseline 2 data are based on posttreatment $I$ for subjects 1 and 2.

${ }^{\mathrm{c} A}$ vs. B: $P<0.05$; B vs. C: NS; D vs. E: $P<0.025$; E vs. F: $P<0.001$; B and E vs. C and F: $P<0.001$.

\subsection{Data analysis}

An analysis of variance and planned comparisons were computed comparing agitation scores during the light treatment periods with scores during the baseline and posttreatment follow-up periods.

\section{Results}

Subjects tended to comply with the protocol, although a staff person always stayed with the sub-

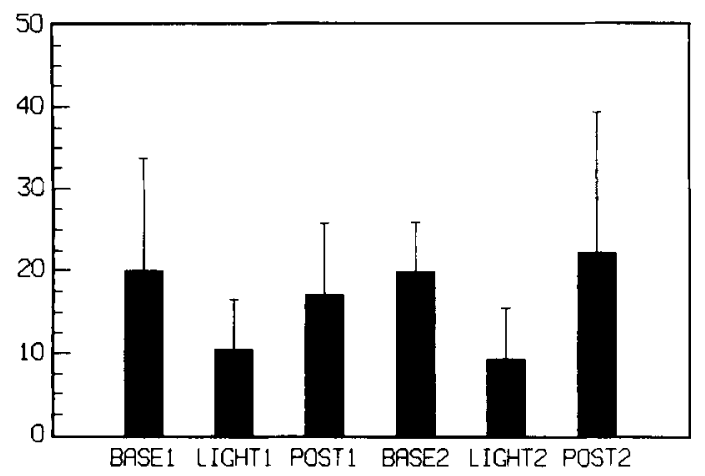

Fig. 1. Mean agitation scores for six subjects. BASEI = baseline 1; LIGHT1 = light treatment 1 ; POST $1=$ posttreatment follow-up 1; BASE2 = baseline 2; LIGHT2 = light treatment 2; POST2 = posttreatment follow-up 2. Bar lines indicate the standard deviation. ject during the light treatment. Most subjects were not generally bothered by the bright light, although some mentioned that it was too bright. Nevertheless, each subject was able to complete the 2-hour daily protocol.

The analysis of variance revealed significant differences between conditions $(F=3.20 ; d f=1,25$; $P<0.025)$. Table 2 lists each subject's agitation scores. The scores refer to the subject's cumulative score on the ABRS over the 4 hours of observations, averaged across days for each (ABABA) week. Fig. 1 illustrates group means over time.

A planned comparison indicated that agitation scores were significantly lower on light treatment days than on nontreatment days ( $F=14.40$; $d f=1,25 ; P<0.001$. Additional contrasts, with the exception of the light treatment 1 vs. posttreatment follow-up 1, were also significant (see Table 2).

\section{Discussion}

The present study supports the hypothesis that light treatment is effective in reducing agitated behaviors. The trend was similar in all six subjects - a reduction of agitated behavior during the light treatment and a return to baseline levels when lights were removed. Although the light effect was significant, it was not lasting. Each subject reacted differently when the bright lights were removed. 
All but subject 3 rebounded by day 2 of posttreatment follow-up 1. The light treatment effect was stronger during the second light trial than during the first light trial for four subjects.

These data do not clarify the potential mechanisms underlying the agitation. Only $4 \mathrm{~h}$ of the 24-h day were sampled, leaving it unclear whether agitation was shifted to another time of day. Future studies will be necessary to increase understanding of these mechanisms.

Subjects were specifically recruited by staff members to participate in an ongoing sleep study of which this study was a part. Agitation was not a requirement for inclusion in the sleep study. All sleep study subjects who were assigned to a morning bright light treatment group and who had any level of agitation were included. The cost of additional equipment and staff necessary for a separate study with different eligibility criteria was beyond the reach of this study and resulted in the low number of subjects studied.

Subjects with low scores reacted differently than subjects with high scores. The more severely agitated subjects (1,2, and 5) appeared to achieve greater reductions in agitated behavior during light treatment than the least agitated subjects, some of whose scores actually increased during treatment. The higher the initial agitation, the lower the agitated behavior with exposure to light, and the more immediate the rebound of agitated behaviors. The present study, while able to address the question of the effectiveness of bright light treatment, could not resolve why highly agitated subjects responded to light and slightly agitated subjects did not. Findings suggest that the highly agitated subjects could be characterized as sundowners because the clinical ratings were done between 16:00 and 20:00 h, and that the other, possibly non-sundowning subjects may be more highly agitated at other times of day. Future studies will be necessary to determine the answer. It is also possible that for the more initially distressed subjects, improvements were more feasible and measurable. The lack of response in slightly agitated subjects may reflect a floor effect that was not measurable. The slightly agitated subjects may have had an adverse reaction to bright light and, if so, should not be included in future studies. The findings support the hypothesis that bright light treatment for subjects experiencing moderate-tosevere agitation does, in fact, result in improvement.

Subjects expressed their agitation almost daily, each displaying a unique pattern. This pattern of idjosyncratic behavior supports the findings of Cohen-Mansfield et al. (1990) that agitation in the demented elderly results from a physical or mental condition and is expressed differently depending on the individual's problem.

Subjects 3 and 6 were medicated with a psychotropic drug (haloperidol) throughout the protocol, and both had increased agitated behavior during the first light trial. However, both had reduced agitation when lights were reintroduced. If and how the use of drugs could differentially affect a subject's response to light treatment needs to be examined.

Relatively few studies are carried out in the institutionalized elderly, whose unique problems make research difficult. During the course of the present study, there were deaths and hospitalizations, prescriptions of psychotropic medications, deaths of loved ones, and even such mundane, but possibly relevant events as bedsores and dehydration, all of which could have affected daily agitation scores. No corrections for those explainable sources of agitation were made, as there was no method to determine their impact. It is possible that the effects of light treatment would have been still more robust if we had corrected for the extreme scores that were possibly independent of the study.

Agitated persons are a problem not only to themselves, but also to the people who live with them and have to cope with the disturbance. Bright light treatment need not be restricted to nursing home residents. Nighttime disruptions, wandering, and yelling are frequent reasons cited for making the decision to put a family member into skilled nursing care (Sanford, 1975; Pollack and Perlick, 1991). The social environment in the home could be improved during the critical evening hours when disruption usually occurs. If the present findings are confirmed in future studies, families might use daily light treatment at home. By decreasing or shifting agitated behaviors, it 
might be possible for the institutionalization of loved ones to be postponed.

\section{Acknowledgments}

The research reported was supported by grants from the National Institute of Aging (AG-08415 and AG-02711) and by the Research Service of the Veterans Affairs Medical Center. Appreciation is owed to the patients, staff, and administration of the San Diego Hebrew Homes and to Denise W. Jones and the University of California, San Diego, sleep studies staff for help with data collection. This study was submitted in partial fulfillment of the requirements for Barbara B. Lovell's Ph.D. Parts of these data were presented at the Annual Meetings of the Association of Professional Sleep Societies and the Society for Light Treatment and Biological Rhythms.

\section{References}

Aronson, M., Cox, D., Guastadisegni, P., Frazier, C., Sherlock, L., Grower, R., Barbera, A., Sternberg, M., Breed, J. and Koren, M.J. (1992) Dementia and the nursing home: association with care needs. $J$ Geriatr Soc 40, 27-33.

Bliwise, D.L., Carroll, J.S. and Dement, W.C. (1989a) Apparent seasonal variation in sundowning behavior in a skilled nursing facility. Sleep Res 18, 408 (abstract).

Bliwise, D.L., Carroll, J.S., Lee, K.A., Nekich, J.C. and Dement, W.C. (1993) Sleep and "sundowning" in nursing home patients with dementia. Psychiatry Res 48, 277-292.

Bliwise, D.L., Lee, K., Carroll, J. and Dement, W. (1989b) A rating scale for assessing sundowning in nursing home patients. Sleep Res 18, 408 (abstract).

Cameron, O. (1941) Studies in senile nocturnal delirium. Psychiatr $Q$ 15, 47-53.

Chesrow, E.J., Kaplitz, S.E., Sabatini, R., Vetra, H. and Marquardt, G.H. (1965) A new psychotherapeutic agent effective in management of anxiety, depression, and behavioral reactions. $J$ Am Geriatr Soc 34, 449-454.

Cohen-Mansfield, J. (1986) Agitated behaviors in the elderly: II. preliminary results in the cognitively deteriorated. $J \mathrm{Am}$ Geriatr Soc 34, 722-727.

Cohen-Mansfield, J. and Billig, N. (1986) Agitated behaviors in the elderly. J Am Geriatr Soc 34, 711-721.
Cohen-Mansfield, J., Marx, M.S. and Rosenthal, A.S. (1989a) A description of agitation in a nursing home. $J$ Gerontol 44, M77-M84.

Cohen-Mansfield, J., Marx, M.S. and Rosenthal, A.S. (1990) Dementia and agitation in nursing home residents: how are they related? Psychol Aging 5, 3-8.

Cohen-Mansfield, J., Watson, V., Meade, W., Gordon, M., Leatherman, J. and Emor, C. (1989b) Does sundowning occur in residents of an Alzheimer's unit? Int $J$ Geriatr Psychiatry 4, 293-298.

Evans, L.K. (1987) Sundown syndrome in institutionalized elderly. J Am Geriatr Soc 35, 101-108.

Fisher, R., Blair, M., Shedletsky, R., Lundell, A., Napoliello, M. and Steinberg, S. (1983) An open dose finding study of melperone in treatment of agitation and irritability associated with dementia. Can J Psychiatry 28, 193-195.

Granacher, R.P. Jr. (1982) Agitation in the elderly: an often treatable manifestation of acute brain syndrome. Postgrad Med 72, 83-95.

Hozumi, S., Okawa, M., Mishima, K., Hishikawa, Y., Hori, H. and Takahashi, K. (1990) Phototherapy for elderly patients with dementia and sleep-wake rhythm disorders - a comparison between morning and evening light exposure. $J p n J$ Psychiatry Neurol 44, 813-814.

Jackson, M.E., Drugovich, M.L., Stenberg, J., Fretwell, M. and Spector, W. (1987) The role of resident characteristics and social support in the presentation of disruptive behaviors in the nursing home. Gerontologist 27, 33 (abstract).

Jeste, D.V. and Krull, A.J. (1991) Behavioral problems associated with dementia: Diagnosis and treatment. Geriatrics 46 , 28-34.

Okawa, M., Mishima, K., Shimizu, T., Iijima, S., Hishikawa, Y., Hozumi, S. and Hori, H. (1989) Sleep-waking rhythm disorders and their phototherapy in elderly patients with dementia. Jpn J Psychiatry Neurol 43, 293-295.

Pollack, C.P. and Perlick, D. (1991) Sleep problems and institutionalization of the elderly. $J$ Geriatr Psychiatry Neurol 4, 204-210.

Sanford, J.R.A. (1975) Tolerance of debility in elderly dependents by supporters at home: its significance for hospital practice. $\mathrm{Br}$ Med $J 3,471-473$.

Satlin, A., Volicer, L., Ross, V., Herz, L. and Campbell, S. (1992) Bright light treatment of behavioral and sleep disturbances in patients with Alzheimer's disease. Am J Psychiatry 149, 1028-1032.

Wild, K., Aronson, M. and Yatzkan, E. (1987) Agitation in Alzheimer's disease: description and measurement. Geronrologist 27, 225 (abstract). 